\title{
Acrosin activity of canine spermatozoa as an index of cellular damage
}

\author{
D. P. Froman, R. P. Amann, P. M. Riek and T. T. Olar \\ Animal Reproduction Laboratory, Colorado State University, Fort Collins, Colorado 80523, U.S.A.
}

\begin{abstract}
Summary. Eight experiments were performed to validate an extraction technique for canine acrosin and to quantitate the acrosin activity of fresh and frozen-thawed spermatozoa. Acrosin activity from fresh spermatozoa differed amongst dogs and was influenced by the interval since previous ejaculation. Freezing and thawing spermatozoa induced a loss of acrosin activity that differed with the extender in which the spermatozoa were frozen. The assay of acrosin activity, in conjunction with motility estimates, provides a more complete evaluation of the efficacy of seminal extenders in attenuating freezing injury than do motility estimates alone.
\end{abstract}

\section{Introduction}

Due to the infrequency of oestrus and man's inability to induce ovulation consistently in the bitch, fertility trials with frozen-thawed canine spermatozoa are extremely expensive and timeconsuming. Even with semen containing $35-40 \%$ progressively motile spermatozoa after thawing, pregnancy rates with frozen-thawed spermatozoa at Colorado State University (R. A. Bowen, T. T. Olar \& B. W. Pickett, unpublished data) have been only $40 \%$ of that obtained by artificial insemination of fresh spermatozoa. Therefore, we postulated that an assay capable of detecting sperm damage, other than the absence of motility, would expedite research with cryopreserved canine spermatozoa by obviating the need of inseminating spermatozoa frozen under less than optimal conditions.

We studied acrosin (EC 3.4.21.10) because it is believed that it enables spermatozoa to penetrate the zona pellucida (Mann \& Lutwak-Mann, 1981b), exists within the acrosome as a zymogen, proacrosin, that is sensitive to cellular damage (Mann \& Lutwak-Mann, 1981a), and is well-defined biochemically (Parrish \& Polakoski, 1979). Furthermore, Goodpasture, Zavos, Cohen \& Zaneveld (1981b) found that the acrosin content of human spermatozoa was reduced by cryopreservation. Since no correlation was found between post-thaw acrosin activity and motility, Goodpasture et al. (1981b) proposed that acrosin assays could serve as an adjunct to estimates of sperm motility. Additionally, Pace, Sullivan, Eliott, Graham \& Coulter (1981), using frozenthawed bull spermatozoa, found a positive, albeit modest, correlation between fertility and the acrosin activity of spermatozoa inseminated.

Canine acrosin could not be extracted consistently by published methodologies (see Goodpasture, Polakoski, \& Zaneveld, 1980). We therefore validated an extraction technique for canine acrosin and used the technique to evaluate cellular damage in cryopreserved canine spermatozoa. 


\section{General Methods}

The pre-sperm and sperm-rich fractions of an ejaculate were collected (Seager \& Fletcher, 1972). Sperm concentration was determined with a spectrophotometer. The percentage of progressively motile spermatozoa was estimated by phase-contrast microscopy $(\times 400)$ at $37^{\circ} \mathrm{C}$ after mixing $6-8$ $\mu \mathrm{l}$ of semen $1: 1$ with an egg yolk-Tris extender $(0.2 \mathrm{M}$-Tris, $70 \mathrm{~mm}$-citric acid, $45 \mathrm{~mm}$-glucose, $3 \%$ (v/v) glycerol and $20 \%$ (v/v) egg yolk plus 500 i.u. penicillin and $1 \mathrm{mg}$ streptomycin per $\mathrm{ml}$ ).

An extraction procedure, with an intra-assay coefficient of variation (CV) of $4 \%$, was developed. A volume of neat semen containing $200 \times 10^{6}$ spermatozoa was layered on $0.5 \mathrm{ml} 50$ mM-benzamidine (B6506, Sigma Chemical Co., St Louis, MO) in $1 \mathrm{M}$-sucrose within a $1.5 \mathrm{ml}$ Eppendorf centrifuge tube and centrifuged for $4 \mathrm{~min}$ in an Eppendorf Model 5414 microcentrifuge $(15600 \mathrm{~g})$. Spermatozoa were separated from seminal plasma and washed by centrifugation through $1 \mathrm{M}$-sucrose. The seminal plasma and sucrose supernatants were discarded, and the pelleted spermatozoa were re-suspended by vortexing in $1 \mathrm{ml}$ of extraction medium. Based upon differential interference-contrast microscopy $(\times 1000),>90 \%$ of the pelleted spermatozoa had an intact acrosome when re-suspended in $0.15 \mathrm{M}-\mathrm{NaCl}$. The extraction medium was $0.3 \mathrm{M}-\mathrm{NaCl}, 50 \mathrm{~mm}$ benzamidine, $0.075 \%(\mathrm{v} / \mathrm{v})$ Hyamine 2389 (gift from Rohm and Haas, Philadelphia, PA), and $0.075 \%(\mathrm{v} / \mathrm{v})$ Triton $\mathrm{X}-100$, in $1 \mathrm{mM}-\mathrm{HCl}(\mathrm{pH} 3)$. The sperm suspension was sonicated for $15 \mathrm{sec}$ at $100 \mathrm{~W}$. Cellular debris was sedimented at $15600 \mathrm{~g}$ and $0.9 \mathrm{ml}$ of the supernatant was transferred to a $1.5 \times 5 \mathrm{~cm}$ column of Sephadex G-25 equilibrated with $1 \mathrm{mM}-\mathrm{HCl}$. The enzyme-rich eluate was collected in a plastic vial. Multiple samples were desalted simultaneously by using columns selected on the basis of equivalent void volumes and recoveries of bovine trypsin (T1005, Sigma; $50 \mu \mathrm{g} / \mathrm{ml} 1$ mM-HCl). Mean recovery for 10 columns was $93 \%$ with a $\mathrm{CV}$ of $2.3 \%$.

Preliminary research showed that the autoactivation of canine proacrosin was completely inhibited by $6 \mathrm{mM}^{-\mathrm{Ca}^{2+}}$. Therefore, a $100-\mu 1$ sample of extract was mixed with $0.5 \mathrm{ml} 0.25 \mathrm{M}$ triethanolamine (T1377, Sigma) at pH 8.0 in a polystyrene cuvette and incubated for $20 \mathrm{~min}$ at $25^{\circ} \mathrm{C}$ (Text-fig. 1) to allow autoactivation of proacrosin. Since acrosin activity was enhanced by the

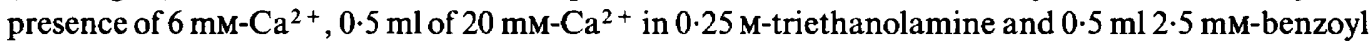
arginine $p$-nitroanilide (BAPNA; B4875, Sigma) were added after autoactivation. After mixing, the linear change in absorbance at $405 \mathrm{~nm}$ and $37^{\circ} \mathrm{C}$ was recorded for $1 \mathrm{~min}$. A molar extinction coefficient of $9950 \mathrm{M}^{-1} \mathrm{~cm}^{-1}$ (Burck \& Zimmerman, 1980) was used to convert the change in absorbance to $\mu$ mol BAPNA hydrolysed. A value of 1 i.u. acrosin activity was defined as the

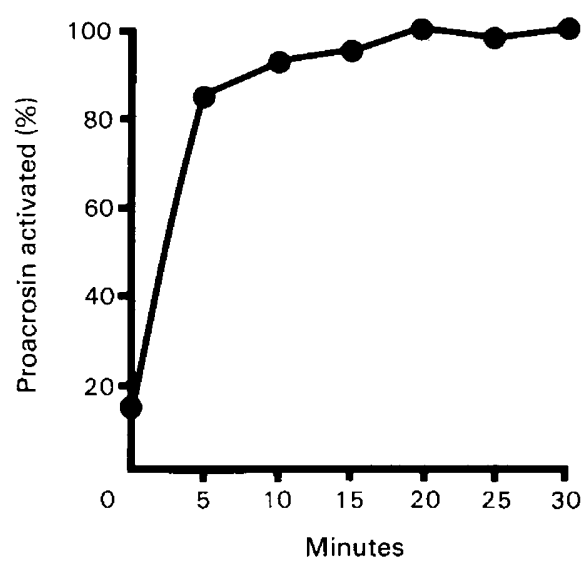

Text-fig. 1. Autoactivation of canine proacrosin at $\mathrm{pH} 8$ and $25^{\circ} \mathrm{C}$. Acrosin was extracted from spermatozoa procured from a pool of 3 ejaculates. Each point represents the mean of 3 replicates. 
amount of enzyme necessary to hydrolyse $1 \mu \mathrm{mol}$ BAPNA/min at $37^{\circ} \mathrm{C}$. The percentage of proacrosin was calculated from baseline and maximal acrosin activities, i.e. before and after the 20min incubation (Goodpasture et al., 1980).

\section{Experimental Methods and Results}

\section{Experiment 1}

The effects of centrifugation and exposure of spermatozoa to egg yolk on the extraction of acrosin were evaluated. A randomized complete block design was used; ejaculates from 6 dogs were used as blocks. Each ejaculate was divided so that $200 \times 10^{6}$ spermatozoa were (a) extracted immediately; (b) centrifuged for $10 \mathrm{~min}$ at $700 \mathrm{~g}$, resuspended in seminal plasma and extracted; or (c) centrifuged but resuspended in egg yolk-Tris before extraction. Data on percentage of proacrosin (after arcsine transformation) and acrosin activity (i.u. $/ 10^{9}$ spermatozoa) were analysed by analysis of variance.

Mean percentage of proacrosin and acrosin activity were not altered by centrifugation of spermatozoa for $10 \mathrm{~min}$ at $700 \mathrm{~g}$ followed by suspension in seminal plasma or egg-yolk-Tris (Table 1). While acrosin activity ranged from 96 to $156 \mathrm{i} . \mathrm{u} . / 10^{9}$ spermatozoa and differed $(P<0.05)$ amongst dogs, percentages of proacrosin were similar.

Table 1. Effects of centrifugation or centrifugation and extension of dog spermatozoa in egg yolk-Tris (EYT)

\begin{tabular}{lcc}
\hline Treatment & $\begin{array}{c}\text { Proacrosin } \\
(\%)\end{array}$ & $\begin{array}{c}\text { Acrosin activity } \\
\text { (i.u./109 }\end{array}$ \\
\hline Control & $89 \pm 1.3$ & $132 \pm 10.7$ \\
Centrifuged control & $90 \pm 1.2$ & $134 \pm 13.5$ \\
Extended in EYT & $88 \pm 1.6$ & $136 \pm 11.5$ \\
\hline
\end{tabular}

Values are mean \pm s.e.m. for 6 replicates.

\section{Experiment 2}

Interassay variability was determined from a pool of 5 ejaculates. Semen was centrifuged 10 $\min$ at $700 \mathrm{~g}$. The spermatozoa were re-suspended at $400 \times 10^{6} / \mathrm{ml}$ in egg yolk-Tris, cooled to $5^{\circ} \mathrm{C}$ over $1 \mathrm{~h}$ and packaged in $0.5-\mathrm{ml}$ French straws. Straws were kept at $5^{\circ} \mathrm{C}$ for $1 \mathrm{~h}$, placed in liquid nitrogen vapour for $20 \mathrm{~min}$, and stored for 1 week in liquid nitrogen. Seven straws were immersed independently in $35^{\circ} \mathrm{C}$ water for $30 \mathrm{sec}$. Each sperm suspension was extracted independently using a different batch of reagents. The inter-assay $\mathrm{CV}$ was $8 \%$.

\section{Experiment 3}

A suitable range of sperm numbers for acrosin extraction was determined. Semen from 6 dogs was pooled and centrifuged. Spermatozoa were resuspended in egg yolk-Tris at 200,300, 400 and $500 \times 10^{6} / \mathrm{ml}$. Three $1-\mathrm{ml}$ samples were extracted per concentration. Units of acrosin activity and percentages of proacrosin were plotted as functions of the number of spermatozoa extracted and linear plots $(r=0.99)$ were obtained (Text-fig. 2). The percentage of proacrosin $(88 \%)$ was independent of the number of spermatozoa extracted (Text-fig. 2). Since the number of spermatozoa available for any experiment was limited, we extracted $200-300 \times 10^{6}$ spermatozoa per sample but kept the number of spermatozoa extracted constant within an experiment. 


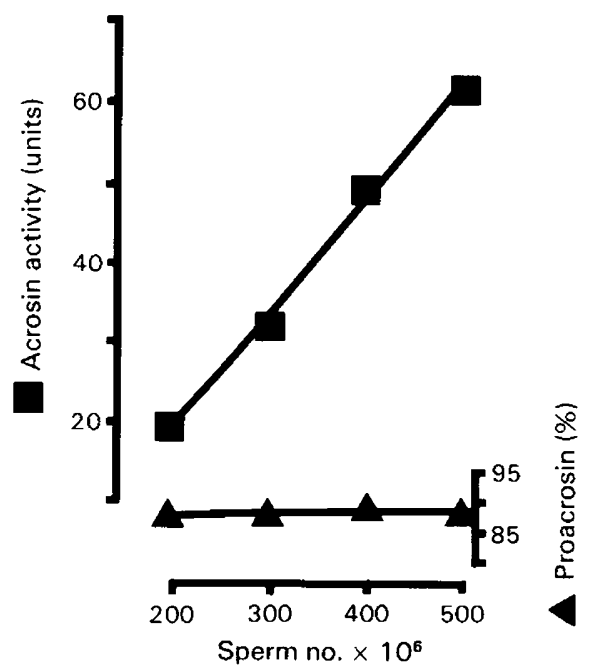

Text-fig. 2. Acrosin activity and percentage of proacrosin as functions of the number of dog spermatozoa extracted. Each point represents the mean of 3 replicates. The s.e.m. values were $\leq 2$ units and $1.4 \%$.

\section{Experiment 4}

The efficiency of the one-step extraction technique was tested. Spermatozoa, $300 \times 10^{6}$ from each of 8 ejaculates, were extracted 3 times, rather than once, as previously outlined. Acrosin activity was undetectable $(<0 \cdot 1$ i.u. $)$ in the third extract. The initial extraction of $300 \times 10^{6}$ spermatozoa removed $90.0 \pm 1.4 \%$ of the activity recovered in the three extractions.

\section{Experiment 5}

The hypothesis that extractable acrosin is proportional to the number of spermatozoa with an intact acrosome before extraction was tested. A pool of semen from 8 dogs was split into two aliquots that were centrifuged. Spermatozoa were resuspended at $500 \times 10^{6} / \mathrm{ml}$ in $0.15 \mathrm{M}-\mathrm{NaCl}$ without or with $50 \mathrm{~mm}$-benzamidine. The former suspension was sonicated for $1.75 \mathrm{~min}$ at $100 \mathrm{~W}$ to lyse acrosomes. By mixing proportions of sonicated and unsonicated suspensions, samples were prepared that theoretically had $0,33,66$ and $99 \%$ intact acrosomes. The percentage of spermatozoa with an intact acrosome was determined in quadruplicate stained smears (Wells \& Awa, 1970) of each sample. Acrosin liberated from the sonicated spermatozoa was removed during the initial centrifugation through sucrose. Acrosin activity was determined in triplicate and plotted against mean percentage of intact acrosomes. Extractable acrosin activity was directly proportional to the number of spermatozoa with an intact acrosome (Text-fig. 3) before extraction.

\section{Experiment 6}

This evaluated whether the variability of acrosin activity amongst ejaculates within dogs was reduced by frequent ejaculation. Seven sexually-rested dogs were ejaculated daily for 16 days. Spermatozoa from ejaculates collected on Days 1, 4, 8 and 16 were extracted. Due to failure of some dogs to ejaculate $\geq 300 \times 10^{6}$ spermatozoa on prescribed days, complete data were obtained from only 3 dogs. 


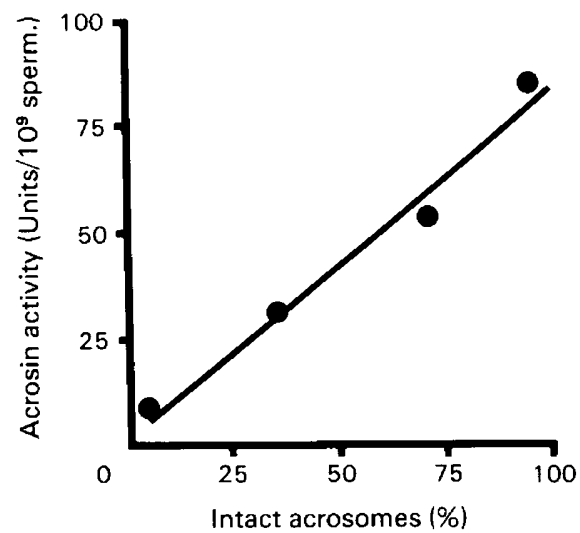

Text-fig. 3. Acrosin activity as a function of the percentage of dog spermatozoa with an intact acrosome $(r=0.97)$. Intact and sonicated spermatozoa were mixed to provide suspensions that theoretically had $0,33,66$ or $99 \%$ intact acrosomes. The actual percentage of spermatozoa with an intact acrosome was calculated and plotted.

Daily semen collection caused the number of spermatozoa per ejaculate to decline (Text-fig. 4). Concomitantly, mean acrosin activity per $10^{9}$ spermatozoa doubled. The percentage of progressively motile spermatozoa remained constant and averaged $82 \%$. Mean percentage of proacrosin also increased since the slope was greater than zero $(P<0.01)$. The large standard error of the mean for acrosin activity on Day 1 corroborated the variability amongst dogs observed in Exp. 1. Variability in mean acrosin activity per $10^{9}$ spermatozoa was reduced but not eliminated.

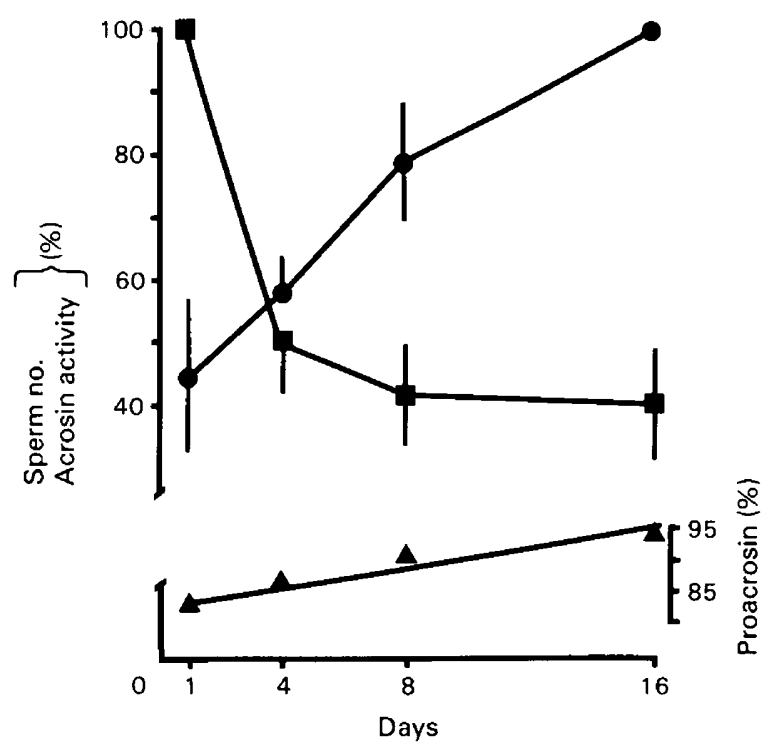

Text-fig. 4. Mean ( \pm s.e.m.) relative number of dog spermatozoa per ejaculate $(\square)$, relative acrosin activity $(\boldsymbol{O})$, and percentage of proacrosin $(\Delta)$ in semen from 3 dogs ejaculated daily after 1 week of sexual rest. Total spermatozoa per ejaculate and acrosin activity per $10^{9}$ spermatozoa are normalized as a percentage of their maximal values. The s.e.m. values for percentage of proacrosin were $\leq 4 \%$. The percentage of progressively motile spermatozoa $(82 \%)$ was independent of day of collection. 


\section{Experiment 7}

The effects of cooling and freezing spermatozoa on acrosin activity and percentage of proacrosin were tested. One ejaculate from each of 9 dogs was centrifuged and the spermatozoa were resuspended at $400 \times 10^{6} / \mathrm{ml}$ in egg yolk-Tris. An aliquant containing $200 \times 10^{6}$ spermatozoa was extracted immediately after re-suspension. The remaining sperm suspension was cooled to $5^{\circ} \mathrm{C}$ over $1 \mathrm{~h}$ and another aliquant was extracted. A third aliquant was packaged, frozen and thawed (as above) before extraction. Data were analysed as outlined for Exp. 1. Comparisons between means were made by the least significant difference test $(P<0.05)$. After suspension in egg yolk-Tris, cooling spermatozoa to $5^{\circ} \mathrm{C}$ reduced their content of proacrosin $(P<0.05)$ without loss of total acrosin activity (Table 2$)$. Freezing and thawing, however, reduced $(P<0.05)$ total activity.

Table 2. Effects of cooling to $5^{\circ} \mathrm{C}$ and of freezing and thawing dog spermatozoa extended in egg yolk-Tris

\begin{tabular}{lcc}
\hline Treatment & $\begin{array}{c}\text { Proacrosin } \\
(\%)\end{array}$ & $\begin{array}{c}\text { Acrosin activity } \\
\text { (i.u. } / 10^{9} \text { spermatozoa) }\end{array}$ \\
\hline Control & $86 \pm 1 \cdot 5^{\mathrm{a}}$ & $92 \pm 6.5^{\mathrm{a}}$ \\
Cooled & $80 \pm 1 \cdot 7^{\mathrm{b}}$ & $79 \pm 5.9^{\mathrm{a}}$ \\
Frozen-thawed & $76 \pm 1 \cdot 4^{\mathrm{b}}$ & $58 \pm 5 \cdot 2^{\mathrm{b}}$ \\
\hline
\end{tabular}

Values are mean \pm s.e.m. for 9 replicates.

Means within columns bearing different superscripts are different $(P<0.05)$.

\section{Experiment 8}

The abilities of two extenders to minimize freezing damage were compared. Semen pooled from 10 dogs was split into two aliquots and centrifuged. Spermatozoa were resuspended at $500 \times 10^{6} / \mathrm{ml}$ in egg yolk-Tris or caprogen extender (New Zealand Dairy Board, 1972). The concentrations of glycerol and egg yolk were $3 \%(\mathrm{v} / \mathrm{v})$ and $20 \%(\mathrm{v} / \mathrm{v})$, respectively, in each extender. Packaging, freezing, and thawing were performed as above. Ten replicates were extracted per extender. Data were analysed as in Exp. 7.

Post-thaw motility of spermatozoa frozen in caprogen extender (Table 3) was $62 \%$ of that of spermatozoa frozen in egg yolk-Tris, but acrosin activity was only $40 \%$ of that of spermatozoa frozen in egg yolk-Tris. Proacrosin was undetectable in extracts of spermatozoa frozen in caprogen extender whereas $69 \%$ of the enzyme extracted from spermatozoa frozen in egg yolk-Tris was proacrosin.

Table 3. Characteristics of dog spermatozoa frozen in egg yolk-Tris (EYT) or caprogen extender

\begin{tabular}{lccc}
\hline Extender & $\begin{array}{c}\text { Motility } \\
(\%)\end{array}$ & $\begin{array}{c}\text { Proacrosin } \\
(\%)\end{array}$ & $\begin{array}{c}\text { Acrosin activity } \\
\text { (i.u./10 }\end{array}$ \\
\hline EYT & $39 \pm 1 \cdot 6^{\mathrm{a}}$ & $69 \pm 2 \cdot 3$ & $55 \pm 2 \cdot 4^{\mathrm{a}}$ \\
Caprogen & $24 \pm 1 \cdot 4^{\mathrm{b}}$ & n.d. & $22 \pm 0.8^{\mathrm{b}}$ \\
\hline
\end{tabular}

Values are mean \pm s.e.m. for 10 replicates; n.d. $=$ not detectable.

Means within columns bearing different superscripts are different $(P<0.01)$. 


\section{Discussion}

While developing an assay for canine acrosin, we found a wide range in acrosin activity per $10^{9}$ spermatozoa, e.g. 3 ejaculates from one dog had activities of 194,55 and 128 i.u. $/ 10^{9}$ spermatozoa. We considered whether the range in acrosin activity was due, in part, to the variable interval spermatozoa spent within the cauda epididymidis and ductus deferens because the interval of abstinence ranged from 2 days to 2 weeks. We tested the hypothesis by sexually resting 7 dogs and then ejaculating them daily to equalize and minimize the interval that spermatozoa spent within the cauda epididymidis and ductus deferens.

Collection of semen for 16 consecutive days had no effect on sperm motility, but resulted in a doubling of mean acrosin activity per $10^{9}$ spermatozoa (Text-fig. 4). The decline in total spermatozoa per ejaculate resulted from a depletion of extragonadal sperm reserves (Olar, Amann $\&$ Pickett, 1984) and presumably was accompanied by a reduction of transit time through the cauda epididymidis from $4 \cdot 5$ days to about $2 \cdot 2$ days (Davies, 1982). We postulate that sperm senescence, undetectable by motility estimates but indicated by loss of acrosin, begins while spermatozoa are within the cauda epididymidis and ductus deferens. This hypothesis is tenable because (a) the cauda epididymidis and ductus deferens are sperm storage sites in the dog (Davies, 1982; Olar et al., 1984), (b) acrosin activity was influenced by the interval since previous ejaculation, and (c) acrosin is an auto-proteolytic enzyme (Müller-Esterl \& Fritz, 1981). Thus, while spermatozoa are within the cauda epididymidis and ductus deferens during long intervals of abstinence, acrosin may form from proacrosin and then undergo autolysis. An alternative hypothesis is that the amount of acrosin inhibitor present in seminal plasma and absorbed to spermatozoa declines with increased frequency of ejaculation.

Comparatively, the percentage of enzyme extracted as proacrosin, $80-94 \%$, was similar to that in acid extracts of human (Goodpasture et al., 1980) and guinea-pig (Goodpasture, Reddy \& Zaneveld, 1981a) spermatozoa. The time required for complete autoactivation of canine proacrosin (Text-fig. 1) was approximately twice that required for human proacrosin at $\mathrm{pH} 8.0$ and $25^{\circ} \mathrm{C}$. As shown for human proacrosin (Goodpasture et al., 1980), the autoactivation of canine proacrosin was inhibited by $\mathrm{Ca}^{2+}$.

Cooling spermatozoa to $5^{\circ} \mathrm{C}$ reduced the percentage of proacrosin but not total acrosin activity (Table 2). Presumably, cooling induced the autoactivation of some proacrosin, but the acrosin that was formed remained within the acrosome. Freezing and thawing did not further reduce the percentage of proacrosin below the level induced by cooling, but total acrosin activity was reduced. Since extractable acrosin activity was proportional to the percentage of spermatozoa with an intact acrosome before extraction (Text-fig. 3), we assume that the acrosomes of some spermatozoa were lysed by freezing and thawing. These observations support the concept (Morris \& Clarke, 1981) that cooling and freezing injuries are distinct and should not be confounded in cryopreservation studies.

The divergence in percentage of progressively motile spermatozoa, percentage of proacrosin and acrosin activity between spermatozoa frozen in egg yolk-Tris and caprogen extender (Table 3) is evidence that few spermatozoa frozen in caprogen extender were functional after thawing. Guinea-pig spermatozoa lose approximately $50 \%$ of their total acrosin activity at the time of the acrosome reaction, but the residual enzyme is about $90 \%$ proacrosin (Goodpasture et al., 1981a). Since proacrosin was undetectable in extracts of spermatozoa frozen in caprogen extender, proacrosin may have autoactivated within intact acrosomes.

Percentages of proacrosin and acrosin activity are indices of cellular damage for canine spermatozoa. However, these values probably are not accurate estimates of fertilizing ability. When spermatozoa frozen in egg yolk-Tris were incubated with canine primary oocytes in canine capacitation medium (Mahi \& Yanagimachi, 1978), the zona of only 5\% of the oocytes was penetrated whereas with fresh spermatozoa $70 \%$ of zonae were penetrated (120 oocytes for each treatment; C. A. Province \& P. M. Riek, unpublished data). In Exp. 7, 63\% of the original acrosin 
activity remained after freezing and thawing. The depression of the rate of zona penetration cited above is greater than would be predicted on the basis of a $37 \%$ reduction in acrosin activity. Alterations in capacitation or a lost ability of spermatozoa to recognize the zona pellucida may explain this discrepancy. Attributes of spermatozoa after freezing and thawing determined by single point assays can detect cellular damage and probably can correctly identify samples likely to have low fertilizing ability. However, false positive predictions probably cannot be eliminated because any test or group of tests fails to account for the sequence of events entailed in fertilization.

This research was partly supported by a grant from the American Kennel Club through the Morris Animal Foundation.

\section{References}

Burck, P.J. \& Zimmerman, R.E. (1980) The inhibition of acrosin by sterol sulphates. J. Reprod. Fert. 58, 121125.

Davies, P.R. (1982) A study of spermatogenesis, rates of sperm production, and methods of preserving the semen of dogs. Ph.D. thesis, University of Sydney.

Goodpasture, J.C., Polakoski, K.L. \& Zaneveld, L.J.D. (1980) Acrosin, proacrosin and acrosin inhibitor of human spermatozoa: extraction, quantitation and stability. J. Androl. 1, 16-27.

Goodpasture, J.C., Reddy, J.M. \& Zaneveld, L.J.D. (1981a) Acrosin, proacrosin and acrosin inhibitor of guinea pig spermatozoa capacitated and acrosomereacted in vitro. Biol. Reprod. 25, 44-55.

Goodpasture, J.C., Zavos, P.M., Cohen, M.R. \& Zaneveld, L.J.D. (1981b) Effects of various conditions of semen storage on the acrosin system of human spermatozoa. J. Reprod. Fert. 63, 397-405.

Mahi, C.A. \& Yanagimachi, R. (1978) Capacitation, acrosome reaction and egg penetration by canine spermatozoa in a simple defined medium. Gamete Res. 1, 101-109.

Mann, T. \& Lutwak-Mann, C. (1981a) Biochemistry of spermatozoa: chemical and functional correlations in ejaculated semen, andrological aspects. In Male Reproductive Function and Semen, pp. 195-268. Springer-Verlag, Berlin.

Mann, T. \& Lutwak-Mann, C. (1981b) Male reproductive function and the composition of semen: General considerations. In Male Reproductive Function and Semen, pp. 1-37. Springer-Verlag, Berlin.

Morris, G.J. \& Clarke, A. (1981) Nomenclature. In Effects of Low Temperature on Biological Membranes, p. xi. Eds G. J. Morris \& A. Clark. Academic Press, London.

Müller-Esterl, W. \& Fritz, H. (1981) Sperm acrosin. Methods in Enzymol. 80, 621-632.

New Zealand Dairy Board (1972) 48th Farm Product Report.

Olar, T.T., Amann, R.P. \& Pickett, B.W. (1984) Relationships among testicular size, daily production and output of spermatozoa and extragonadal spermatozoal reserves of the dog. Biol. Reprod. (in press).

Pace, M.M., Sullivan, J.J., Eliott, F.I., Graham, E.F. \& Coulter, G.H. (1981) Effects of thawing temperature, number of spermatozoa and spermatozoal quality on fertility of bovine spermatozoa packaged in $.5-\mathrm{ml}$ French straws. J. Anim. Sci. 53, 693-701.

Parrish, R.F. \& Polakoski, K.L. (1979) Mammalian sperm proacrosin-acrosin system. Int. J. Biochem. 10, 391-395.

Seager, S.W.J. \& Fletcher, W.S. (1972) Collection, storage, and insemination of canine semen. Lab. Anim. Sci. 22, 177-182.

Wells, M.E. \& Awa, O.A. (1970) New technique for assessing acrosomal characteristics of spermatozoa. J. Dairy Sci. 53, 227-232.

Received 21 June 1983 